\title{
Hierarchic Euclidean Skeletons in Cubical Complexes ${ }^{\star}$
}

\author{
Michel Couprie \\ Université Paris-Est, LIGM, Équipe A3SI, ESIEE Paris, France \\ m. couprie@esiee.fr
}

\begin{abstract}
In the 90s, several authors introduced the notion of a hierarchic family of 2D Euclidean skeletons, evolving smoothly under the control of a filtering parameter. We provide in this article a discrete framework which formalizes and generalizes this notion, in particular to higher dimensions. This framework allows us to propose a new skeletonization scheme and to prove several important properties, such as topology preservation and stability w.r.t. parameter changes.
\end{abstract}

Despite the simplicity of the most common definition of a skeleton, as the set of all centers of maximal included balls, its use in real applications often raises difficult problems. One of the main issues is its lack of stability.

In 2005, F. Chazal and A. Lieutier introduced the $\lambda$-medial axis [4, a particular class of filtered skeletons, and studied its properties, in particular those related to stability. A major outcome of 4 is the following property: informally, except for particular values of the filtering parameter, the $\lambda$-medial axis remains stable under perturbations of the shape that are small with regard to the Hausdorff distance. However, the original definition of the $\lambda$-medial axis only holds and makes sense in the (continuous) Euclidean $n$-dimensional space. In $[3$, J. Chaussard et al. introduced the definition of a discrete $\lambda$-medial axis in $\mathbb{Z}^{n}$, and showed that it provides good robustness to boundary noise and invariance by rotation in practice.

For certain applications however, the $\lambda$-medial axis cannot be used because, when a small modification of the filtering parameter $\lambda$ is done around some particular values, topological changes or abrupt appearing/disappearing of branches typically occur in the resulting skeketon.

On the other hand, several authors have proposed a general idea that leads to the notion of a family of skeletons, evolving smoothly under the control of a single parameter. They called it hierarchic skeletons [10, veinerization [11] or multiscale skeletons [8]. Such a family of skeletons can be summarized by a function, called potential residual in [10. The skeletons are obtained as level sets (i.e., thresholds) of this function. The method of R.L. Ogniewicz and O. Kübler [10] applies to $2 \mathrm{D}$ shapes that are (sets of) planar polygons in $\mathbb{R}^{2}$, and the resulting skeleton is a set of straight line segments which do not necessarily

\footnotetext{
* This work has been partially supported by the "ANR BLAN07-2_184378 MicroFiss" project.
} 
have their extremities on a grid. The authors give a proof that the obtained skeleton is homotopy-equivalent to the original shape, but discretizations of these skeletons in $\mathbb{Z}^{2}$ do not generally share the same property. Veinerization [11] and multiscale skeletons [8] are methods that operate in the 2D square grid, and that are based on the same general idea as Ogniewicz and Kübler's. However 11] does not provide an algorithm to compute skeletons in practice, and the algorithm proposed in [8] does not guarantee topology preservation. Extensions of these works to higher-dimensional spaces (e.g., 3D) have not been considered so far, to our knowledge.

In this article, we propose an effective and flexible method for building and filtering discrete skeletons, in the framework of cubical complexes. We ensure topology preservation by founding our method on the collapse operation, an elementary deformation that preserves the homotopy type.

First, we propose a thinning procedure that combines three ideas: $\lambda$-medial axis (Sec. 2), directional parallel thinning, and guided thinning (Sec. 3). This procedure also produces a sequence of collapse operations from which we derive an acyclic graph structure that we call a flow graph.

Based on this flow graph, we define the notion of topological map. We prove that if $M$ is a topogical map on an object $X$, then any level set of $M$ has the same homotopy type as $X$ (Th. 5 ). Moreover, we prove that if the real numbers $a$ and $b$ are close to each other, then the shapes $M_{a}$ and $M_{b}$ (the level sets of $M$ at levels $a$ and $b$ ) are close to each other 1 with respect to the Hausdorff distance (Th. 6). This property will assess the stability of our final skeletonization method w.r.t. the parameter value.

Then, we show how to build particular topological maps based on different measures of shape characteristics, thanks to the notion of upstream (Sec. 3 , Sec. 6).

Finally, we propose a skeletonization scheme that produces families of filtered homotopic skeletons (Sec. 7). A filtered skeleton is obtained as a level set of the obtained topological map.

Proofs of the stated properties, experimental evaluation, and more illustrations can be found in an extended version of this article [6].

\section{Cubical Complexes and Collapse}

In this section, we recall briefly some basic definitions on cubical complexes, see also [1] for more details.

Let $S$ be a set. If $T$ is a subset of $S$, we write $T \subseteq S$. We denote by $|S|$ the number of elements of $S$.

Let $\mathbb{Z}$ be the set of integers. We consider the families of sets $\mathbb{F}_{0}^{1}, \mathbb{F}_{1}^{1}$, such that $\mathbb{F}_{0}^{1}=\{\{a\} \mid a \in \mathbb{Z}\}, \mathbb{F}_{1}^{1}=\{\{a, a+1\} \mid a \in \mathbb{Z}\}$. A subset $f$ of $\mathbb{Z}^{n}, n \geqslant 2$, which is the Cartesian product of exactly $m$ elements of $\mathbb{F}_{1}^{1}$ and $(n-m)$ elements of $\mathbb{F}_{0}^{1}$ is called a face or an $m$-face in $\mathbb{Z}^{n}, m$ is the dimension of $f$, we write $\operatorname{dim}(f)=m$.

\footnotetext{
${ }^{1}$ In the sense of Lipschitz continuity.
} 


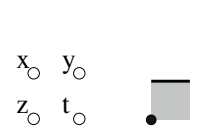

(a)

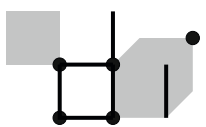

(c)

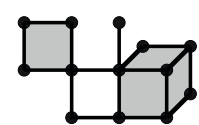

(d)

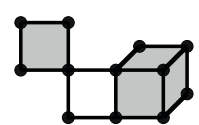

(e)

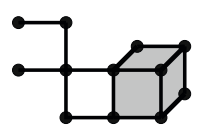

(f)

Fig. 1. (a) Four points in $\mathbb{Z}^{2}: x=(0,1) ; y=(1,1) ; z=(0,0) ; t=(1,0)$. (b) A graphical representation of the set of faces $\left\{f_{0}, f_{1}, f_{2}\right\}$, where $f_{0}=\{z\}=\{0\} \times\{0\}$ (a 0 -face), $f_{1}=\{x, y\}=\{0,1\} \times\{1\}$ (a 1-face), and $f_{2}=\{x, y, z, t\}=\{0,1\} \times\{0,1\}$ (a 2-face). (c) A set of faces that is not a complex. (d) A set of faces that is a complex. (e,f) Two steps of elementary collapse from (d).

We denote by $\mathbb{F}^{n}$ the set composed of all faces in $\mathbb{Z}^{n}$. An $m$-face is called a point if $m=0$, a (unit) edge if $m=1$, a (unit) square if $m=2$, a (unit) cube if $m=3$.

Let $f$ be a face in $\mathbb{F}^{n}$. We set $\hat{f}=\left\{g \in \mathbb{F}^{n} \mid g \subseteq f\right\}$ and $\hat{f}^{*}=\hat{f} \backslash\{f\}$. Any $g \in \hat{f}$ is called a face of $f$.

A finite set $X$ of faces in $\mathbb{F}^{n}$ is a complex (in $\mathbb{F}^{n}$ ) if for each face $f \in X$, we have $\hat{f} \subseteq X$. See in Fig. 1)(d,e,f) some examples of complexes, and in Fig. 1 (b,c) examples of sets of faces that are not complexes.

The collapse operation is an elementary topology-preserving transformation which has been introduced by J.H.C. Whitehead [14] and plays an important role in combinatorial topology. It can be seen as a discrete analogue of a continuous deformation (a strong deformation retract). Collapse is known to preserve the homotopy type.

Let $X$ be a complex in $\mathbb{F}^{n}$ and let $(f, g) \in X^{2}$. If $f$ is the only face of $X$ that strictly includes $g$, then $g$ is said to be free for $X$ and the pair $(f, g)$ is said to be a free pair for $X$. Notice that, if $(f, g)$ is a free pair, then we have necessarily $\operatorname{dim}(g)=\operatorname{dim}(f)-1$.

Let $X$ be a complex, and let $(f, g)$ be a free pair for $X$. Let $m=\operatorname{dim}(f)$. The complex $X \backslash\{f, g\}$ is an elementary collapse of $X$, or an elementary $m$-collapse of $X$ (see Fig. 1, (e) is an elementary 1-collapse of (d), (f) is an elementary 2-collapse of (e)).

Let $X, Y$ be two complexes. We say that $X$ collapses onto $Y$, and we write $X \searrow Y$, if $Y=X$ or if there exists a collapse sequence from $X$ to $Y$, i.e., a sequence of complexes $\left\langle X_{0}, \ldots, X_{\ell}\right\rangle$ such that $X_{0}=X, X_{\ell}=Y$, and $X_{i}$ is an elementary collapse of $X_{i-1}$, for each $i \in\{1, \ldots, \ell\}$. Let $J=\left\langle\left(f_{i}, g_{i}\right)\right\rangle_{i=1}^{\ell}$ be the sequence of pairs of faces of $X$ such that $X_{i}=X_{i-1} \backslash\left\{f_{i}, g_{i}\right\}$, for any $i \in\{1, \ldots, \ell\}$. We also call the sequence $J$ a collapse sequence (from $X$ to $Y$ ).

\section{The Discrete $\lambda$-Medial Axis and the Projection Radius Map}

The original definition of the $\lambda$-medial axis (see [4]) holds and makes sense in the (continuous) Euclidean $n$-dimensional space. The definition of a discrete $\lambda$-medial axis (DLMA) in $\mathbb{Z}^{n}$ is given in [3], together with an experimental evaluation of its stability and rotation invariance. 

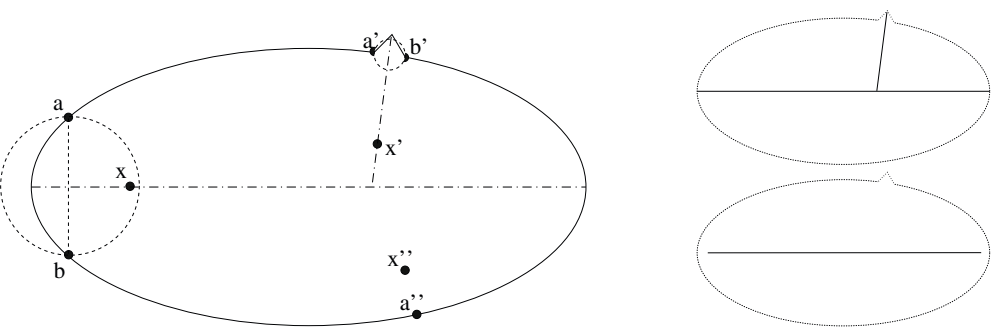

Fig. 2. Illustration of the $\lambda$-medial axis. Left: Points $x, x^{\prime}$ and $x^{\prime \prime}$ and their respective closest boundary points. Top right: $\lambda$-medial axis with $\lambda=\epsilon$, a very small positive real number. Bottom right: $\lambda$-medial axis with $\lambda=d\left(a^{\prime}, b^{\prime}\right)+\epsilon$.

Notice that the DLMA applies on a digital image (i.e., a subset of $\mathbb{Z}^{n}$ ), not on a complex. However, the bijective correspondance between elements of $\mathbb{Z}^{n}$ and $n$-faces in $\mathbb{F}^{n}$ allows us to use the DLMA and related notions in the context of cubical complexes.

Let $x=\left(x_{1}, \ldots, x_{n}\right), y=\left(y_{1}, \ldots, y_{n}\right) \in \mathbb{R}^{n}$, we denote by $d(x, y)$ the Euclidean distance between $x$ and $y$, in other words, $d(x, y)=\left(\sum_{k=1}^{n}\left(y_{k}-x_{k}\right)^{2}\right)^{\frac{1}{2}}$. Let $S \subseteq \mathbb{R}^{n}$, we set $d(y, S)=\min _{x \in S}\{d(y, x)\}$.

Let $x \in \mathbb{R}^{n}, r \in \mathbb{R}^{+}$, we denote by $B_{r}(x)$ the ball of radius $r$ centered on $x$, defined by $B_{r}(x)=\left\{y \in \mathbb{R}^{n} \mid d(x, y) \leqslant r\right\}$.

Let $S$ be a nonempty subset of $\mathbb{R}^{n}$, and let $x \in \mathbb{R}^{n}$. The projection of $x$ on $S$, denoted by $\Pi_{S}(x)$, is the set of points $y$ of $S$ which are at minimal distance from $x$; more precisely,

$$
\Pi_{S}(x)=\{y \in S \mid \forall z \in S, d(y, x) \leqslant d(z, x)\} .
$$

Let $X$ be an open bounded subset of $\mathbb{R}^{n}$, and let $\lambda \in \mathbb{R}^{+}$. We denote by $\bar{X}$ the complement set of $X$, i.e., $\bar{X}=\mathbb{R}^{n} \backslash X$. The $\lambda$-medial axis of $X$ is the set of points $x$ in $X$ such that the radius of the smallest ball that includes $\Pi_{\bar{X}}(x)$ is not less than $\lambda$ (see Fig. 2).

For each point $x \in \mathbb{Z}^{n}$, we define the direct neighborhood of $x$ as $N(x)=\{y \in$ $\left.\mathbb{Z}^{n} \mid d(x, y) \leqslant 1\right\}$.

Transposing directly the definition of the $\lambda$-medial axis to the discrete grid $\mathbb{Z}^{n}$ would yield unsatisfactory results (see [3]), this is why we need the following notion. Let $S \subseteq \mathbb{Z}^{n}$, and let $x \in S$. The extended projection of $x$ on $\bar{S}$ (where $\left.\bar{S}=\mathbb{Z}^{n} \backslash S\right)$, denoted by $\Pi_{\frac{e}{S}}(x)$, is the union of the sets $\Pi_{\bar{S}}(y)$, for all $y$ in $N(x)$ such that $d(y, \bar{S}) \leqslant d(x, \bar{S})$.

Let $S$ be a finite subset of $\mathbb{Z}^{n}$, and let $\lambda \in \mathbb{R}^{+}$. We define the function $P R_{S}$ which associates, to each point $x$ of $S$, the value $P R_{S}(x)$ that is the radius of the smallest ball enclosing all the points of the extended projection of $x$ on $\bar{S}$. In other terms, $P R_{S}(x)=\min \left\{r \in \mathbb{R}^{+} \mid \exists y \in \mathbb{R}^{n}, \Pi \frac{e}{S}(x) \subseteq B_{r}(y)\right\}$, and we call $P R_{S}(x)$ the projection radius of $x$ (for $S$ ). The discrete $\lambda$-medial axis of $S$, denoted by $D L M A(S, \lambda)$, is the set of points $x$ in $S$ such that $P R_{S}(x) \geqslant \lambda$.

Note that the function $P R_{S}$ can be computed once and stored as a grayscale image, and that any DLMA of $S$ is a level set of this function at a particular 
value $\lambda$. Notice also that DLMA has not, in general, the same topology as the original shape. For more details, illustrations and performance analysis, see [3].

\section{Guided Collapse and Flow Graph}

In this section, we introduce a thinning scheme that produces a collapse sequence, based on an arbitrary priority map (e.g., a distance map or a projection radius map). The general idea of guided thinning is not new: it has been used by several authors to produce skeletons based on the Euclidean distance [7/312, and consists of using the priority function in order to specify which elements must be considered at each step of the thinning. Here, we combine this general idea with a parallel directional collapse algorithm introduced in [2], in order to minimize the number of arbitrary decisions. When several elements share the same priority, which may occur quite often, we remove in parallel all such elements that satisfy a condition based on direction and dimension. All directions and dimensions are successively explored.

First, we need to define the direction of a free face. Let $X$ be a complex in $\mathbb{F}^{n}$, let $(f, g)$ be a free pair for $X$. Since $(f, g)$ is free, we know that $\operatorname{dim}(g)=$ $\operatorname{dim}(f)-1$, and it can be easily seen that $f=g \cup g^{\prime}$ where $g^{\prime}$ is the translate of $g$ by one of the $2 n$ vectors of $\mathbb{Z}^{n}$ with all coordinates equal to 0 except one, which is either +1 or -1 . Let $v$ denote this vector, and $c$ its non-null coordinate. We define $\operatorname{Dir}(f, g)$ as the index of $c$ in $v$, it is the direction of the free pair $(f, g)$. Its orientation is defined as $\operatorname{Orient}(f, g)=1$ if $c=+1$, and as $\operatorname{Orient}(f, g)=0$ otherwise.

Now, we are ready to introduce algorithm GuidedCollapse (see Alg. 1). The symbol + is used to denote the action of appending an element at the end of a sequence.

We have the following property.

Proposition 1. Whatever the complex $X$ and the map $P$ from $X$ to $\mathbb{R}, X$ collapses onto GuidedCollapse $(X, P)$.

Algorithm GuidedCollapse may be implemented to run in $O(N \log N)$ time complexity, where $N$ denotes the cardinality of $X$, using a balanced binary tree data structure (see [5]) for representing the set $R$.

To conclude this section, we introduce the notion of a flow graph associated to a given collapse sequence.

A (finite directed) graph is a pair $(V, E)$ where $V$ is a finite set and $E$ is a subset of $V \times V$. An element of $V$ is called a vertex, an element of $E$ is called an arc. A path in $(V, E)$ is a sequence $\left\langle v_{i}\right\rangle_{i=0}^{\ell}$ of vertices such that $\ell \geqslant 0$ and for all $i \in\{1, \ldots, \ell\}$, we have $\left(v_{i-1}, v_{i}\right) \in E$. The number $\ell$ is the length of the path. If $\ell=0$ the path is said trivial. If $v_{0}=v_{\ell}$ the path is a cycle. The graph is acyclic if it does not contain any non-trivial cycle.

Definition 2. Let $X$ be a complex and $J=\left\langle\left(f_{i}, g_{i}\right)\right\rangle_{i=1}^{\ell}$ be a collapse sequence from $X$. For any $k \in\{1, \ldots, \ell\}, \ell \geqslant 0$, we set $X_{k}=X \backslash\left\{f_{i}, g_{i}\right\}_{i=1}^{k}$. We set $E_{1}=\left\{\left(g_{i}, f_{i}\right)\right\}_{i=1}^{\ell}$ and $E_{2}=\bigcup_{k=1}^{\ell}\left\{\left(f_{k}, g\right) \mid g \in \hat{f}_{k}^{*} \cap X_{k}\right\}$. 


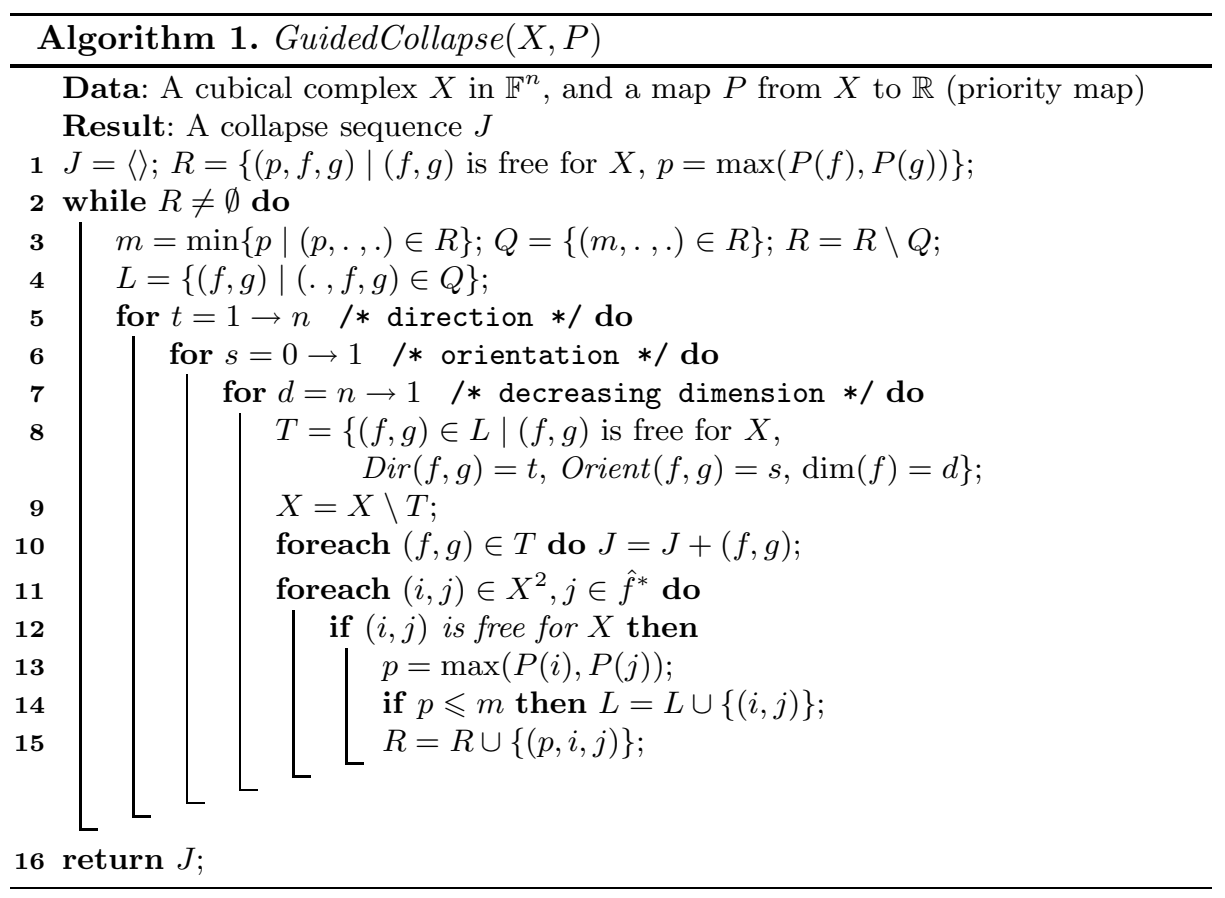

The flow graph associated to $J$ is the (directed) graph whose vertex set is $X$ and whose edge set is $E=E_{1} \cup E_{2}$.

This definition is illustrated in Fig. 3. It can be easily seen that, whatever the complex $X$ and the collapse sequence $J$ from $X$, the flow graph associated to $J$ is acyclic.

\section{Upstream of a Vertex and Its Valuation}

From now, we consider a collapse sequence $J=\left\langle\left(f_{i}, g_{i}\right)\right\rangle_{i=1}^{\ell}$ from a complex $X$, and its associated flow graph $\left(X, E=E_{1} \cup E_{2}\right)$. Using the notations of Def. 2, any pair $\left(f_{k}, g_{k}\right)$ of $J$ is free for $X_{k-1}$, and we have $X=X_{0} \searrow \ldots \searrow X_{\ell}$. We define $F=\left\{f_{i}\right\}_{i=1}^{\ell}, G=\left\{g_{i}\right\}_{i=1}^{\ell}$ and $X_{J}=F \cup G$.

Let $x \in X$, we denote by $\Gamma(x)$ the set of successors of $x$ in the acyclic graph $(X, E)$, that is, $\Gamma(x)=\{y \in X \mid(x, y) \in E\}$, and we denote by $\Gamma^{-1}(x)$ the set of predecessors of $x$ in this graph, that is, $\Gamma^{-1}(x)=\{y \in X \mid(y, x) \in E\}$. We denote by $d^{+}(x)$ the outer degree of the vertex $x$ in the graph $(X, E)$, that is, the number of successors of $x$.

We call upstream of $x$ the set of all vertices that are ancestors of $x$ in the flow graph, that is, the set $U(x)=\{y \in X \mid$ there is a path from $y$ to $x$ in $(X, E)\}$.

In a collapse sequence, certain pairs can be swapped or eliminated, yielding another collapse sequence. Intuitively, the elements of the upstream of a face $x$ of $X$ are those that must indeed be collapsed before $x$ can itself collapse. 

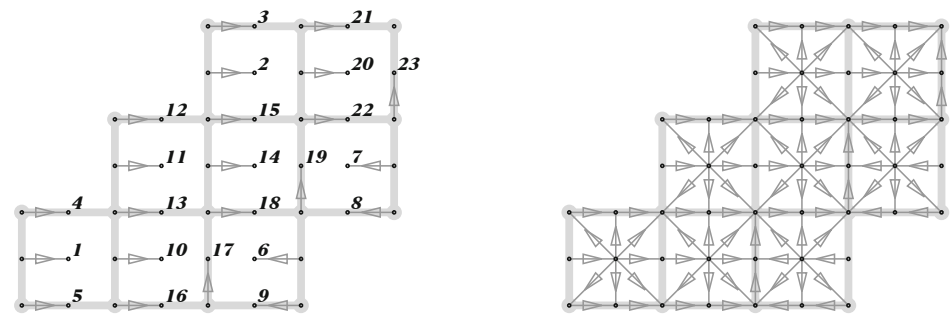

Fig. 3. Left: A collapse sequence $J$. Each pair $\left(f_{i}, g_{i}\right)$ of $J$ is depicted by an arrow from $g_{i}$ to $f_{i}$. The numbers indicate the indices of the pairs in $J$. Right: The flow graph associated to $J$.

Let $L$ be a map from $X$ to $\mathbb{R} \cup\{+\infty\}$. Roughly speaking, the map $\widetilde{L}$ defined below cumulates, for each vertex $x$, the values of $L$ on all vertices of the upstream of $x$.

Definition 3. Let $L$ be a map from $X$ to $\mathbb{R} \cup\{+\infty\}$. We define the map $\widetilde{L}$ such that, for any $x \in X$ :

$$
\widetilde{L}(x)=L(x)+\sum_{y \in \Gamma^{-1}(x)} \widetilde{L}(y) / d^{+}(y)
$$

Notice that this definition is recursive, and that it makes sense since the graph $(X, E)$ is acyclic. Intuitively, the division by $d^{+}(y)$ is motivated by the fact that a value must not be taken in account several times in the sum. The values $\widetilde{L}(x)$ can be computed by a quite simple recursive program (given in 6]) that has a linear time complexity.

Two particularly simple functions $L$ yield meaningful indicators associated to the elements of $X$. Let us first consider the function $L_{1}$ such that $L_{1}(x)=1$ if $\operatorname{dim}(x)=n$, and $L_{1}(x)=0$ otherwise. The map $\widetilde{L}_{1}$ associates, to each element $x$ of $X$, the "area of $U(x)$ " (or its volume in 3D). Now, let us consider $L_{2}=1_{B(X)}$, where $B(X)$ is the set of all faces that are free for $X$. We call $B(X)$ the border of $X$. In other words, $L_{2}(x)=1$ if $x \in B(X)$, and $L_{2}(x)=0$ otherwise. The map $\widetilde{L}_{2}$ associates, to each element $x$ of $X$, a measure (length in $2 \mathrm{D}$, surface area in 3D) of $U(x) \cap B(X)$.

Fig. $4\left(a_{1}, a_{2}\right)$ show the maps $L_{1}$ and $L_{2}$ respectively, for the same object $Y$. The maps $\widetilde{L}_{1}$ and $\widetilde{L}_{2}$ are displayed in Fig. $4\left(b_{1}, b_{2}\right)$.

\section{Topological Maps}

In this section, we introduce the notion of topological map. A topological map based on a collapse sequence $J$ is a map on the elements of $X$ that satisfies certain conditions relative to $J$ and its associated flow graph. Then, we prove an important property of such maps: if $M$ is a topological map, then any level set of $M$ is homotopy-equivalent to $X$. In Sec. 6] we will show how to build such a map, based on any given function on $X$. 


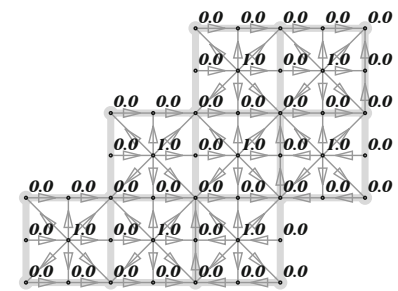

$\left(a_{1}\right)$

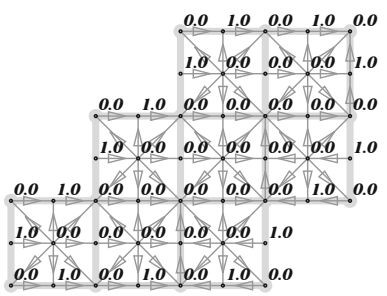

$\left(a_{2}\right)$

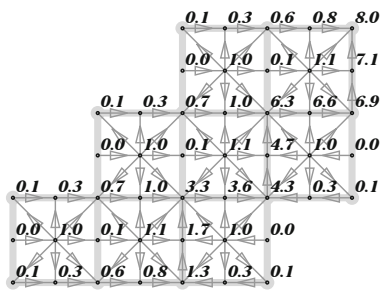

$\left(b_{1}\right)$

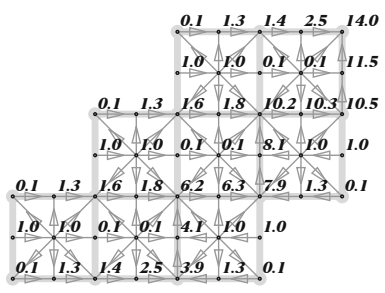

$\left(b_{2}\right)$

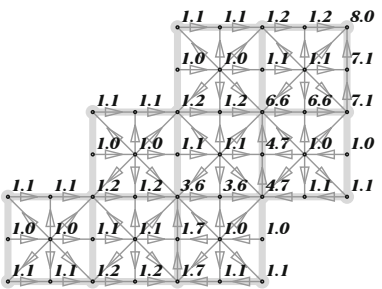

$\left(c_{1}\right)$

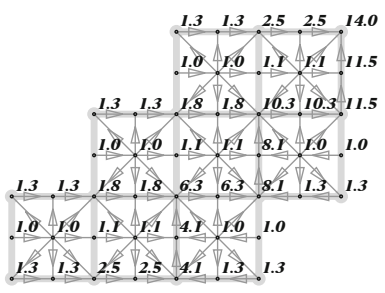

$\left(c_{2}\right)$

Fig. 4. $\left(a_{1}, a_{2}\right)$ Maps $L_{1}$ and $L_{2}$ on the same complex $Y$. $\left(b_{1}, b_{2}\right)$ Maps $\widetilde{L}_{1}$ and $\widetilde{L}_{2}$. For the sake of readability, only one digit after the decimal point is displayed. $\left(c_{1}, c_{2}\right) \alpha$ Topological maps induced by $\widetilde{L}_{1}$ and $\widetilde{L}_{2}$, respectively, with $\alpha=0.1$ (see Sec. 5 and Sec. 6).

Definition 4. Let $M$ be a map from $X$ to $\mathbb{R} \cup\{+\infty\}$. We say that $M$ is a topological map on $X$ (based on $J$ ) if:

i) for all $(g, f)$ in $E_{1}, M(g)=M(f)$; and

ii) for all $(f, g)$ in $E_{2}, M(g)>M(f)$; and

iii) for all $g$ in $X \backslash X_{J}, M(g)=+\infty$.

Let $\alpha$ be a positive real number. If we replace ii) with the stronger requirement: ii') for all $(f, g)$ in $E_{2}, M(g) \geqslant M(f)+\alpha$, then we say that $M$ is an $\alpha$-topological map on $X$ (based on $J$ ).

The notion of topological map is inspired from the one of discrete Morse function (see [9]). A topological map can be seen (apart from the infinite values) as a particular case of discrete Morse function, and Th. 5 could also be proved using results of 9 .

Let $\lambda \in \mathbb{R} \cup\{+\infty\}$, we define $M_{\lambda}=\{x \in X \mid M(x) \geqslant \lambda\}$, the (upper) level set of $M$ at level $\lambda$. The main property of a topological map $M$ is that any level set of $M$ is homotopy-equivalent to $X$, as implied by the following theorem (Th. 5 , see Fig. 5 for an illustration).

Theorem 5. Let $M$ be a topological map on $X$. Whatever the number $\lambda \in$ $\mathbb{R} \cup\{+\infty\}$, the complex $X$ collapses onto $M_{\lambda}$.

The next theorem (Th. 6) expresses the stability of our skeletonization scheme, with respect to the variations of the filtering parameter. 


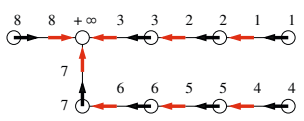

(a)

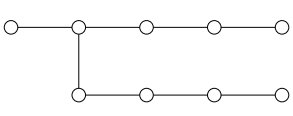

(b)

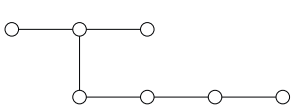

(c)

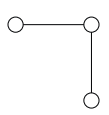

(d)

Fig. 5. (a) A 1-complex $X$, a flow graph on $X$ (black arrows for arcs of $E_{1}$, red arrows for arcs of $E_{2}$ ), and a (1-)topological map $M$ on $X$ (numbers). (b,c,d) Level sets of $M$ at levels 0,3 and 7 , respectively.

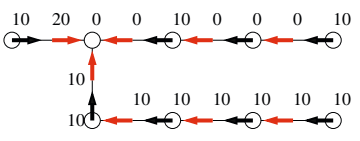

(a)

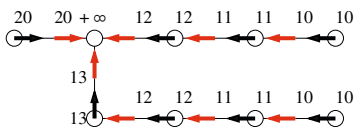

(b)

Fig. 6. (a) A map $L$ on the complex $X$ of Fig. 5. (b) The 1-topological map induced by $L$.

Let $S, T$ be two subsets of $\mathbb{R}^{n}$. We set $H(S \mid T)=\max _{s \in S}\left\{\min _{t \in T}\{d(s, t)\}\right\}$, and $d_{H}(S, T)=\max \{H(S \mid T), H(T \mid S)\}$ is the Hausdorff distance between $S$ and $T$. Let $X$ be a complex in $\mathbb{F}^{n}$, we denote by $\mathcal{S}(X)$ the union of all faces of $X$, called the support of $X$. For comparing two complexes $X$ and $Y$, we consider the Hausdorff distance between their supports $\mathcal{S}(X)$ and $\mathcal{S}(Y)$.

Theorem 6. Let $\alpha \in \mathbb{R}, \lambda \in \mathbb{R} \cup\{+\infty\}, \alpha>0, \lambda \geqslant 0$. Let $k \in \mathbb{N}$. Let $M$ be an $\alpha$-topological map on $X$. Then, $d_{H}\left(\mathcal{S}\left(M_{\lambda}\right), \mathcal{S}\left(M_{\lambda+k \alpha}\right)\right) \leqslant k$.

\section{Topological Map Induced by an Arbitrary Map}

In this section, we show that given any map $L$ on $X$, we can define and compute a topological map that is "close to" $L$, more precisely it is the lowest map above $L$ that is a topological map.

Definition 7. Let $L$ be any map from $X$ to $\mathbb{R} \cup\{+\infty\}$, and let $\alpha$ be a positive real number. We consider a map $M$ such that:

a) $M$ is an $\alpha$-topological map; and

b) for all $f$ in $X_{J}, M(f) \geqslant L(f)$; and

c) $M$ is minimal for conditions a) and b), that is, any map $M^{\prime}$ verifying both a) and b) is such that $M^{\prime} \geqslant M$.

As stated by the following property, $M$ is uniquely defined. We say that the map $M$ is the $\alpha$-topological map induced by $L$.

Proposition 8. Let $M$ and $M^{\prime}$ be two maps that verify conditions a), b) and c) of Def. 7. Then, we have $M=M^{\prime}$.

This notion is illustrated in Fig. 6 and in Fig. $4\left(c_{1}, c_{2}\right)$.

Next, we give an algorithm (Alg. 2) that computes the $\alpha$-topological map induced by any given map on $X$. Before this, let us recall briefly the notions of 

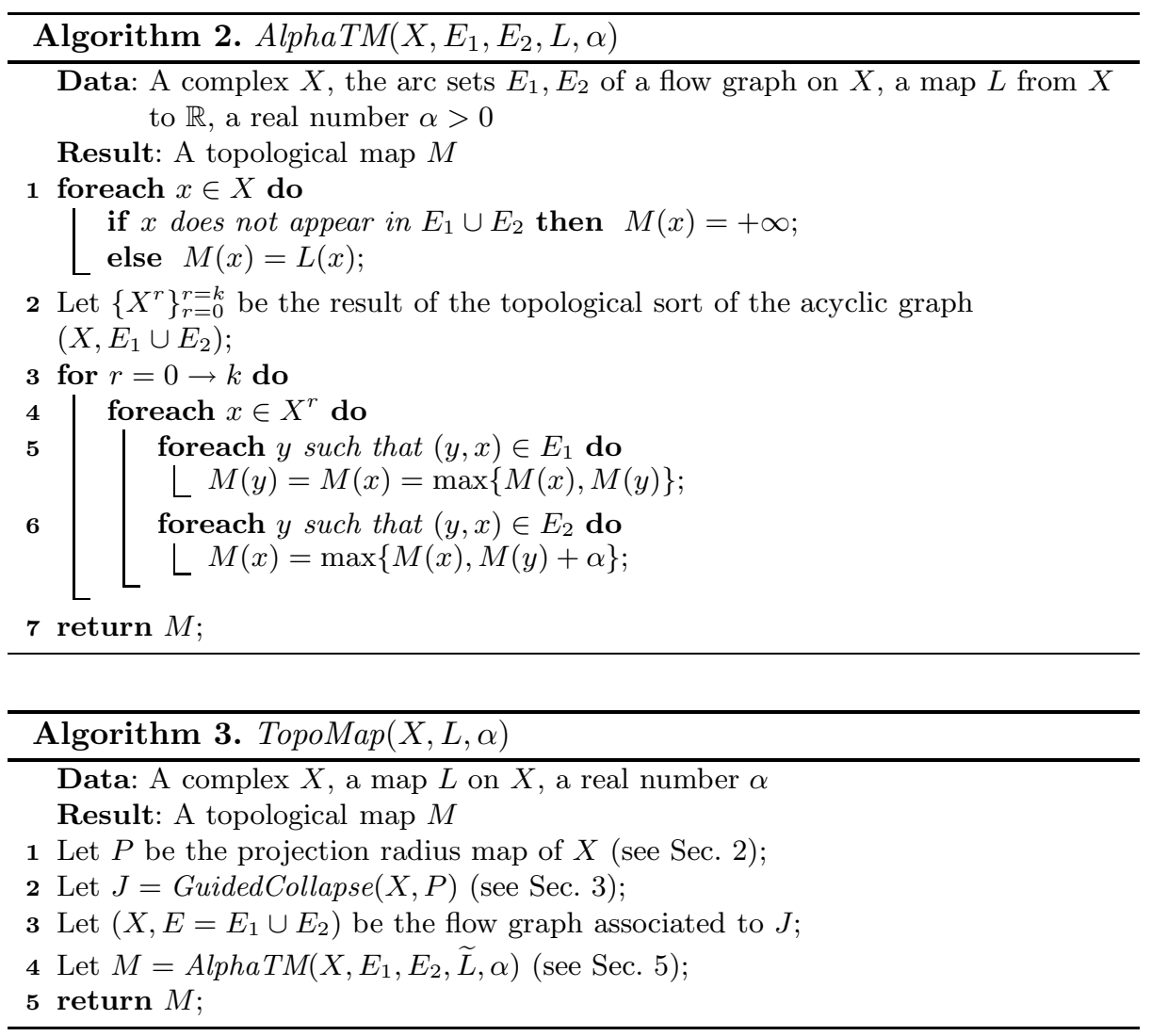

rank and topological sort (an introduction to topological sort, including definition, properties and algorithm, can be found, e.g., in [5]). Let $G=(V, E)$ be an acyclic graph and let $x \in V$, the rank of $x$ in $G$ is the length of the longest path in $G$ that ends in $x$. The topological sort of $G$ is an operation that results in a partition $\left\{V^{r}\right\}_{r=0}^{r=k}$ of $V$ such that each $V^{r}$ is the subset of $V$ containing all vertices of rank $r$.

Proposition 9. Let $L$ be a map from $X$ to $\mathbb{R}$, and let $\alpha$ be real number, $\alpha>0$. The result of $\operatorname{AlphaTM}\left(X, E_{1}, E_{2}, L, \alpha\right)$ is the $\alpha$-topological map induced by $L$.

\section{Computing Hierarchic Skeletons}

Let us now summarize our method to produce families of filtered homotopic skeletons (see algorithm 3). It is assumed here that $X$ is a pure $n$-complex in $\mathbb{F}^{n}$, that is, a complex in which each face is included in an $n$-face.

First, we compute the projection radius map (Sec. 2) on the $n$-faces of $X$, and extend it to the other elements of $X$ (if $y \in X$ is not an $n$-face, then we set $P(y)$ to the max of $P\left(x_{i}\right)$ where the $x_{i}$ 's are all $n$-faces that include $y$ ). 

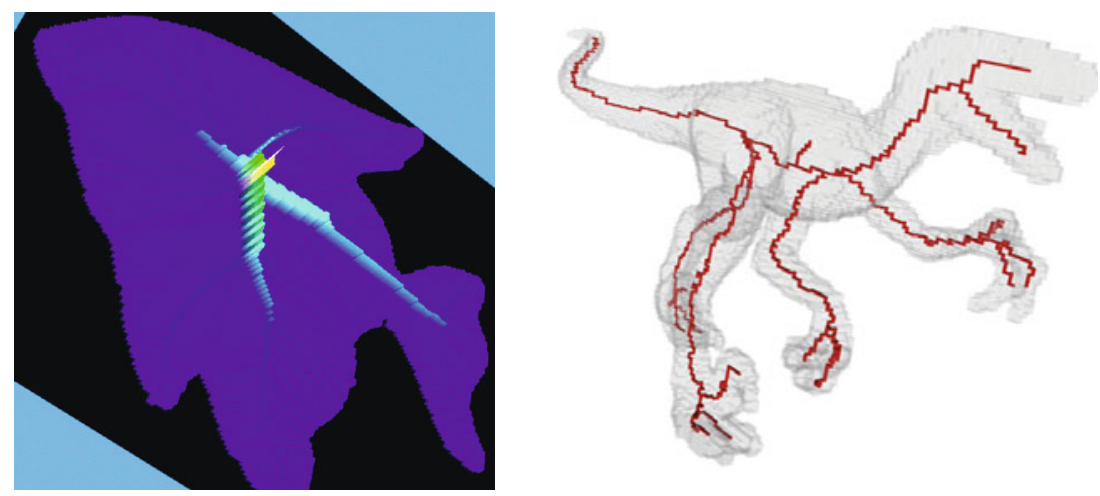

Fig. 7. Left: a rendering of the result of the TopoMap operator, using the map $L_{2}$. Right: a filtered curvilinear skeleton of a $3 \mathrm{D}$ shape.

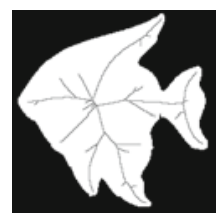

(a)

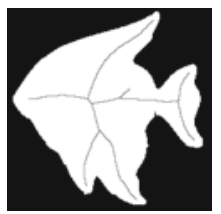

(b)

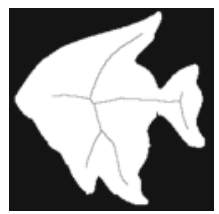

(c)

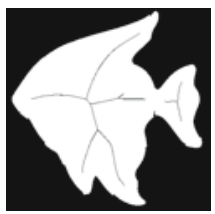

(d)

Fig. 8. (a,b,c) Three level sets of $\operatorname{TopoMap}\left(X, L_{2}\right)$, at values 25, 50 and 130, respectively. (d) DLMA of $X$, with $\lambda=45$.

Using algorithm1 (Sec. 3) we build a collapse sequence and a flow graph on $X$. By construction, the upstream of any vertex $x$ of this flow graph is composed by elements that, in any family of filtered skeletons, should disappear before $x$ does.

Integrating information given by map $L$ allows us to associate, to each element $x$ of $X$, a value $\widetilde{L}(x)$ that represents a measure of the upstream of $x$. The lower this value, the sooner the point $x$ may disappear.

Then, thanks to algorithm 2 (Sec. 5), we produce a topological map $M$ based on this measure. Due to Th. 5] we know that any level set of $M$ is homotopyequivalent to $X$. Therefore, filtered (i.e., pruned) skeletons are obtained by thresholding the map $M$; lowest levels of threshold correspond to highest levels of detail. Some results are shown in Fig. 7 and Fig. 8 .

\section{Conclusion}

The method that we propose is guaranteed to preserve topology and is stable with respect to variations of the filtering parameter, as stated by Th. 5 and Th. 6 respectively. It is designed to work in 3D as well as in 2D. Furthermore, our method is highly flexible: many variants can be imagined, in particular by 
choosing alternative valuations of the upstream. Future work include comparison with other methods for computing discrete filtered Euclidean skeletons.

\section{References}

1. Bertrand, G., Couprie, M.: A new 3D parallel thinning scheme based on critical kernels. In: Kuba, A., Nyúl, L.G., Palágyi, K. (eds.) DGCI 2006. LNCS, vol. 4245, pp. 580-591. Springer, Heidelberg (2006)

2. Chaussard, J., Couprie, M.: Surface thinning in 3D cubical complexes. In: Wiederhold, P., Barneva, R.P. (eds.) IWCIA 2009. LNCS, vol. 5852, pp. 135-148. Springer, Heidelberg (2009)

3. Chaussard, J., Couprie, M., Talbot, H.: Robust skeletonization using the discrete lambda-medial axis. Pattern Recognition Letters, 1-10 (2010) (to appear), doi.org/10.1016/j.patrec.2010.09.002

4. Chazal, F., Lieutier, A.: The lambda medial axis. Graphical Models 67(4), 304-331 (2005)

5. Cormen, T.H., Leiserson, C., Rivest, R.: Introduction to algorithms. MIT Press, Cambridge (1990)

6. Couprie, M.: Topological maps and robust Euclidean skeletons in cubical complexes (2010), http://www.esiee.fr/ coupriem/Pdf/topomaps.pdf

7. Davies, E.R., Plummer, A.P.N.: Thinning algorithms: a critique and a new methodology. Pattern Recognition 14, 53-63 (1981)

8. Falcao, A.X., da Fontoura Costa, L., da Cunha, B.S.: Multiscale skeletons by image foresting transform and its application to neuromorphometry. Pattern Recognition 35(7), 1571-1582 (2002)

9. Forman, R.: Morse theory for cell complexes. Advances in Mathematics 134(1), 90-145 (1998)

10. Ogniewicz, R.L., Kübler, O.: Hierarchic Voronoi skeletons. Pattern Recognition 28(33), 343-359 (1995)

11. Pierrot-Deseilligny, M., Stamon, G., Suen, C.Y.: Veinerization: a new shape description for flexible skeletonization. IEEE Trans. on Pattern Analysis and Machine Intelligence 20(5), 505-521 (1998)

12. Pudney, C.: Distance-ordered homotopic thinning: a skeletonization algorithm for 3D digital images. Comp. Vision and Image Understanding 72(3), 404-413 (1998)

13. Talbot, H., Vincent, L.: Euclidean skeletons and conditional bisectors. In: Procs. VCIP 1992, SPIE, vol. 1818, pp. 862-876 (1992)

14. Whitehead, J.H.C.: Simplicial spaces, nuclei and $m$-groups. Procs. of the London Mathematical Society 45(2), 243-327 (1939) 